\title{
Evaluación del frenillo lingual en neonatos. Una revisión sistemática
}

Evaluation of the lingual frenulum in neonates. A Systematic Review

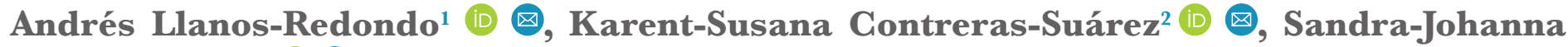 \\ Aguilar-Cañas $^{1}$ (D) Q
}

${ }^{1}$ Departamento Fonoaudiología; Facultad de Salud; Universidad de Pamplona; Pamplona; Colombia.

${ }^{2}$ Centro de Psicología y Terapias IPS; Cúcuta; Colombia.

Correspondencia

Andrés Llanos-Redondo, email:

andres.llanos@unipamplona.edu.co

\section{Gitar así}

Llanos-Redondo, Andrés; Contreras-Suárez, Karent-Susana; Aguilar-Cañas, Sandra-

Johanna. (2021). Evaluación del frenillo lingual en neonatos. Una revisión sistemática. Revista de Investigación e Innovación en Ciencias de la Salud. 3(1), 87-97. https://doi.org/10.46634/riics.71

Recibido: $13-08-2020$

Revisado: 19-03-2021

Aceptado: $23-03-2021$

Editor en jefe

Jorge Mauricio Cuartas Arias, Ph.D. (iD

Coeditor

Fraidy-Alonso Alzate-Pamplona, MSc.

Gopyright $($ 2021. Fundación Universitaria María Cano. La Revista de Investigación e

Innovación en Ciencias de la Salud proporciona acceso abierto a todo su contenido bajo los términos de la licencia Creative Commons Attribution-NonCommercial-NoDerivatives 4.0 International (CG BY-NG-ND 4.0).

Declaración de intereses

Los autores han declarado que no hay conflicto de intereses.

Disponibilidad de datos

Todos los datos relevantes se encuentran en el artículo. Para mayor información, comunicarse con el autor de correspondencia.

\section{Resumen}

Introducción: el objetivo de esta revisión es responder al interrogante: ¿cuál es la metodología más eficaz de evaluación de frenillo lingual en neonatos? Bajo los parámetros de objetividad, claridad y validación.

Métodos: el estudio se realizó a través de una revisión sistemática, llevada a cabo con metodología Cochrane, en la que se utilizaron los descriptores de evaluación, frenillo lingual, anquiloglosia y recién nacido, y ejecutado en los bancos de datos Pubmed, Science Direct, Scielo. Para analizar el nivel de evidencia y grado de recomendación clínica, se tuvo en cuenta la clasificación GRADE y GEBM de Oxford.

Resultados: por medio de la revisión se encontraron 2 evaluaciones y un tamizaje específicamente para recién nacidos, que evalúa de los 0 a los 30 días, los cuales se validan por medio de estudios de especificidad.

Análisis y discusión: se identificó que los principales parámetros para diagnosticar una alteración en el frenillo lingual son la anatomía y su funcionalidad.

Conclusiones: según los estudios encontrados sí existe un método eficaz para el diagnóstico del frenillo lingual en neonatos.

\section{Palabras calve}

Frenillo lingual, anquiloglosia, evaluación, recién nacido, boca, lengua, alteraciones, evidencia clínica

\section{Abstract}

Introduction: the objective of this review is to answer the question: What is the most effective methodology for assessing lingual frenulum in neonates? Under the parameters of objectivity, clarity and validation.

Methods: the study was conducted through a systematic review carried out using the Cochrane methodology, in which the evaluation descriptors, lingual frenulum, 


\section{Financiamiento}

La investigación fue financiada por la Vicerrectoría de Investigaciones de Universidad de Pamplona con código de proyecto número 400-156.012-060 (GA317-BP-2017-FASEII), Acta No 022 del 31 de julio de 2018.

\section{Descargo de responsabilidad}

El contenido de este artículo es responsabilidad exclusiva de los autores y no representa una opinión oficial de sus instituciones ni de la Revista de Investigación e Innovación en Ciencias de la Salud.

\section{Agradecimientos}

Al Departamento de Fonoaudiología de la Universidad de Pamplona. ankyloglossia, and newborn were used, and developed in the Pubmed, Science Direct and Scielo data banks. To analyze the level of evidence and degree of clinical recommendation, the GRADE and CEBM classification of Oxford was taken into account.

Results: through the review we found 2 evaluations and a screening specifically for newborns that evaluates from 0 to 30 days, which are validated through specificity studies.

Analysis and discussion: it was identified that the main parameters to diagnose an alteration in the lingual frenum are its anatomy and functionality.

Conclusions: according to the studies found, there is an effective method for the diagnosis of the lingual frenulum in neonates.

\section{Key words}

Lingual frenulum, ankyloglossia, evaluation, newborn, mouth, tongue, alterations, clinical evidence

\section{Introducción}

El frenillo lingual es un pliegue medio de la membrana mucosa que se extiende desde la superficie posterior de la encía de la lengua, cubriendo la superficie lingual de la cresta alveolar anterior [1], y que tiende a desplazarse a medida que aumenta la dimensión vertical de la boca, produciendo el alargamiento lingual. Este proceso lleva a que el frenillo lingual termine ubicándose en la parte anterior de la lengua [2]. Su formación se origina en etapas embrionarias cuando se desarrollan los músculos hioglosos y geniogloso de la cavidad bucal. En los neonatos, el frenillo lingual se ubica en "la base anterior de la lengua hasta la base del hueso alveolar de la mandíbula" y tras algunas semanas, al alargarse de manera gradual la lengua, este pliegue termina por "ocupar la porción central de la cara ventral de la lengua, la cual será su posición definitiva" [3 p2].

Una alteración del frenillo lingual puede inmovilizar la lengua o restringirla. Su etiología aún es confusa, pero puede estar asociada a factores de riesgo tales como ser varón y una historia familiar positiva a la presencia de alteraciones en este aspecto [4].

Existen diferentes tipos de frenillo lingual, los cuales se pueden clasificar de acuerdo a sus características. Normal, que es la fijación en medio de la cara inferior de la lengua, y en el piso de la boca el frenillo es generalmente visible a partir de las carúnculas sublinguales. Anteriorizado, que es cuando en la cara inferior de la lengua la fijación se encuentra por encima de la porción medial de la estructura. Corto, que es la fijación en el medio de la cara inferior de la lengua como la del frenillo normal, aunque es de menor tamaño. Usualmente, la fijación en el piso de la boca es visible a partir de la cresta alveolar y casi siempre están visibles las puntas de la fijación del frenillo en esta cresta. Corto y anteriorizado es aquel que presenta una combinación de las características del frenillo corto y del anteriorizado y anquiloglosia, en el que la lengua está totalmente fijada al piso de la boca [5].

En algunas ocasiones, cuando se observa una de estas afecciones, se encuentra una lengua con movimientos restringidos, impidiéndole que se proyecte para afuera de la boca o haga contacto con el paladar duro y con la superficie lingual de los dientes antero-superiores, estando la boca abierta. Esto a su vez dificulta la alimen- 
tación, masticación, deglución, articulación del habla, cambios dentales y las funciones sociales, y es ahí cuando se encuentra la necesidad de la intervención quirúrgica en niños en edades tempranas [6].

Por lo anteriormente descrito, este estudio tiene como objetivo encontrar la metodología más eficaz de evaluación de frenillo lingual validada específicamente en neonatos, ya que se considera que debe evitarse cualquier alteración, bien sea por el funcionamiento como en la anatomía de la lengua, que pueda perjudicar el desenvolvimiento de la vida, y para esto la importancia de un diagnóstico claro y objetivo basado en evidencia clínica y medible.

\section{Método}

Se realizó una revisión Cochrane conformada por los siguientes seis pasos [7]:

\section{Definición de los DeCs y los MeSH}

El estudio tuvo en cuenta los descriptores que se presentan en la Tabla 1. La búsqueda y análisis de los descriptores giró en torno a la temática de metodología de evaluación de frenillo lingual en neonatos.

\begin{tabular}{l|l}
\multicolumn{1}{c}{ Tabla 1. Descriptores uthlizados en la revisión sistemática } \\
\hline Frenillo Lingual & \multicolumn{1}{c}{ MeSH } \\
\hline Anquiloglosia & Lingual Frenum \\
\hline Evaluación & Ankyloglossia \\
\hline Tamizaje Masivo & Evaluation \\
\hline Sensibilidad y Especificidad & Mass Screening \\
\hline Recién Nacido & Sensitivity and Specificity \\
\hline Lactante & Infant, Newborn \\
\hline
\end{tabular}

\section{Jerarquías por cada descriptor}

La jerarquía de los descriptores se hizo a partir de las siguientes categorías: Lingual Frenum, Ankyloglossia, Evaluation, Mass Screening, Sensitivity and Specificity, Infant, Newborn.

\section{Establecimiento de cruces de variables}

La revisión contó con tres variables constantes durante la búsqueda que se relacionan en la Tabla 2.

\begin{tabular}{c|c|c}
\multicolumn{2}{c}{ Tabla 2. Gruces de las variables con los MeSH } \\
\hline Métodos & Población de estudio & Condición de la patología \\
\hline Evaluation & Infant, Newborn & Lingual Frenum \\
\hline Mass Screening & Infant & Ankyloglossia \\
\hline Sensitivity and Specificity & & \\
\hline
\end{tabular}




\section{Registro de filtrado}

Los bancos de datos en los que se llevó a cabo la revisión fueron: PubMed, Science Direct y Scielo. El proceso de filtrado en el estudio se llevó a cabo teniendo en cuenta los siguientes criterios de inclusión:

- Tipos de estudio: Ensayos clínicos, estudios de caso, revisiones y revisiones sistemáticas, ensayos clínicos aleatorizados.

- Especie: Humanos.

- Población: Neonatos.

\section{URL de artículos seleccionados}

Es una etapa de la revisión en la que se documentaron uno a uno los cruces y los resultados que se generaron con la respectiva URL que conduce a los textos encontrados.

\section{Ficha de selección de artículos}

En esta etapa se organizó una ficha en la que se ingresaron los artículos que se encuentran en este estudio, con un registro de la información de autores, base de datos, año, título y palabras claves.

\section{Resultados}

Por medio de la revisión Cochrane, se trató de dar respuesta a la pregunta: ¿Cuál es el método más eficaz de evaluación de frenillo lingual en neonatos? Con la que se hallaron los siguientes resultados:

\section{Etapa de identificación}

Con el fin de identificar las metodologías usadas para la evaluación del frenillo lingual en neonatos, y, a su vez, reconocer cuál es la más eficaz, se dio uso de los MeSH a lo largo de la revisión, los cuales se cruzaron permanentemente con el MesH evaluation and lingual frenum. En la Tabla 3 se encuentra el número de artículos arrojados en esta etapa de la búsqueda:

\begin{tabular}{|c|c|c|}
\hline \multicolumn{2}{|r|}{ Cruces } & \multirow{2}{*}{ Artículos } \\
\hline Variable dependiente & Variable independiente & \\
\hline \multirow{6}{*}{ Evaluation } & lingual frenum + infant newborm & 6 \\
\hline & Ankyloglossia + infant newborm & 84 \\
\hline & lingual frenum + straining & 95 \\
\hline & sensitivity and specifity + lingual frenum & 9 \\
\hline & Ankyloglossia + Infant & 257 \\
\hline & lingual frenum + Infant & 260 \\
\hline \multicolumn{2}{|r|}{ Total } & 711 \\
\hline
\end{tabular}




\section{Etapa de Screening}

La búsqueda para este segmento se hizo en los bancos de datos PubMed, ScienceDirect y Scielo. Se aplicaron los filtros correspondientes de la investigación, donde se obtuvieron los resultados que se relacionan en la Tabla 4.

\begin{tabular}{|c|c|c|}
\hline \multicolumn{2}{|r|}{ Cruces } & \multirow{2}{*}{ Artículos } \\
\hline Variable dependiente & Variable independiente & \\
\hline \multirow{7}{*}{ Evaluation } & lingual frenum + infant newborm & 2 \\
\hline & Ankyloglossia + infant newborm & 11 \\
\hline & lingual frenum + straining & 9 \\
\hline & sensitivity and specifity + lingual frenum & 1 \\
\hline & Ankyloglossia + Infant & 14 \\
\hline & lingual frenum + Infant & 12 \\
\hline & Total & 49 \\
\hline
\end{tabular}

\section{Etapa de Elegibilidad}

Teniendo en cuenta los resultados con los criterios de inclusión anteriormente mencionados, se filtraron los artículos a través de los títulos de interés para la revisión, quedando un total de 49 artículos. Los criterios que se tuvieron en cuenta para descartar los artículos fueron:

1. Se repetían los artículos en la base de datos.

2. Los artículos científicos no correspondían a la población objeto de estudio de la investigación.

3. Los artículos no respondían a la pregunta inicial.

4. Algunos artículos no tenían relación con el tema.

\section{Etapa de inclusión}

Los artículos con criterios de elegibilidad fueron organizados en fichas que reportan datos de autores, URL, abstracts de los artículos en su idioma original y resúmenes en español. Para esta revisión, 35 artículos contaron con este proceso.

\section{Etapa de identificación del nivel de evidencia}

A partir del tipo de diseño de los estudios seleccionados, se categorizaron los textos siguiendo para el caso del nivel de evidencia la jerarquía propuesta por CEMB. Además, para definir el grado de recomendación clínica se hizo uso del sistema GRADE. Los artículos seleccionados fueron en su mayoría clasificados según la CEBM en estudios $2^{a}$ Revisión sistemática de estudios de cohortes, con homogeneidad. En la Tabla 5 se observan los ítems que se evalúan en cada una de las pruebas halladas en esta revisión, las cuales debían ser validadas por medio de Hazelbaker assessment tool for lingual frenulum function (ATLFF), Bristol tongue assessment tool (BTAT) y test de la lengüita. 


\section{Tabla 5. Comparación de evaluaciones validadas de frenfllo lingual en neonatos}

\begin{tabular}{|c|c|c|c|}
\hline $\begin{array}{l}\text { Valoraciones de } \\
\text { frenillo lingual } \\
\text { en neonatos }\end{array}$ & $\begin{array}{l}\text { Hazelbaker assessment } \\
\text { tool for lingual frenulum } \\
\text { function }\end{array}$ & $\begin{array}{l}\text { Bristol tongue assessment } \\
\text { tool (btat) }\end{array}$ & La lengüita \\
\hline \multirow{3}{*}{ Historia clínica } & & & 1.Datos personales \\
\hline & & & 2. Antecedentes personales y familiares \\
\hline & & & 3. Lactancia materna \\
\hline \multirow{8}{*}{$\begin{array}{l}\text { Aspecto del } \\
\text { frenillo }\end{array}$} & 1. Apariencia de la lengua & $\begin{array}{l}\text { 1. Aspecto de punta de la } \\
\text { lengua }\end{array}$ & 1. Postura habitual de labios \\
\hline & 2. Elasticidad del frenillo & $\begin{array}{l}\text { 2. Unión en la encía de } \\
\text { inferior }\end{array}$ & $\begin{array}{l}\text { 2. Tendencia de la posición de la lengua } \\
\text { durante el llanto }\end{array}$ \\
\hline & \multirow{2}{*}{$\begin{array}{l}\text { 3. Longitud del frenillo } \\
\text { cuando se eleva la lengua }\end{array}$} & & $\begin{array}{l}\text { 3. Forma de la lengua cuando se eleva } \\
\text { durante el llanto }\end{array}$ \\
\hline & & & \\
\hline & $\begin{array}{l}\text { 4. Unión de frenillo } \\
\text { lingual con la lengua }\end{array}$ & & 4. Frenillo de la lengua \\
\hline & $\begin{array}{l}\text { 5. Unión de frenillo } \\
\text { lingual con el borde } \\
\text { alveolar inferior }\end{array}$ & & 4.1 Espesor del frenillo \\
\hline & & & $\begin{array}{l}\text { 4.2 Fijación del frenillo en la cara sublingual } \\
\text { (ventral) de la lengua }\end{array}$ \\
\hline & & & 4.3 Fijación del frenillo en el piso de la boca \\
\hline \multirow{7}{*}{ Funcionalidad } & 1. Lateralización & $\begin{array}{l}\text { 1. Elevación de lengua con } \\
\text { boca abierta (llorando) }\end{array}$ & $\begin{array}{l}\text { 1. Succión no nutritiva (succión del dedo } \\
\text { meñique con guante) }\end{array}$ \\
\hline & 2. Elevación de la lengua & 2. Protrusión de la lengua & $\begin{array}{l}\text { 2. Succión nutritiva (próxima a la hora de } \\
\text { lactar, observar al bebé lactando durante } 5 \\
\text { minutos) }\end{array}$ \\
\hline & 3. Extensión de la lengua & & 2.1. Ritmo de succión \\
\hline & $\begin{array}{l}\text { 4. Expansión de la parte } \\
\text { anterior de la lengua }\end{array}$ & & $\begin{array}{l}\text { 2.2. Coordinación entre succión/deglución/ } \\
\text { respiración }\end{array}$ \\
\hline & $\begin{array}{l}\text { 5. Ventosas (capacidad de } \\
\text { hacer el vacío) }\end{array}$ & & 2.3. "Muerde" el pezón \\
\hline & 6. Peristalsis & & 2.4. Chasquidos durante la succión \\
\hline & 7. Chasquido & & \\
\hline
\end{tabular}




\section{Análisis y discusión}

La ankiloglosia o lengua atada aún genera debates debido a las pocas herramientas que permiten dar un diagnostico objetivo. Así mismo, la falta de un concepto universal que la defina produce divisiones en el manejo de esta afección. Además, la sintomatología es variable, ya que no todos los recién nacidos dan una señal de la presencia de una alteración en el frenillo lingual. En algunas ocasiones son las madres quienes reportan que existe una dificultad en la [8] movilidad de la lengua de su hijo. No obstante, el número de madres convencidas de que sus hijos presentan esta condición excede el número real [9].

El frenillo se presenta como una membrana mucosa situada bajo la lengua. En el caso de que se encuentre alterado, puede llegar a generar un trastorno del crecimiento y desarrollo, caracterizado por la fijación anormal de la lengua. Esto reduce o impide su movilidad y puede originar efectos adversos anatómicos y/o funcionales en la cavidad bucal [1].

Debido a esto, es importante encontrar herramientas que permitan prevenir disfunciones tanto en el presente como en un futuro. Los criterios clínicos utilizados para diagnosticar la anquiloglosia varían mucho en la literatura. Varios autores [10-12] utilizan criterios basados en las características físicas de la anatomía bucal del paciente [13], pero si se valoran solo los criterios anatómicos y de su función, se encontrará que no todos los frenillos están presentando una alteración [8]. Esto dependerá de si son flexibles, de su punto de inserción, si están formados de tejido fibroso o blando y de si son largos o cortos [14], puesto que algunos pueden parecer cortos, pero tienen suficiente elasticidad para cumplir su función [15].

Las metodologías más usadas para dar un diagnóstico de una alteración del frenillo lingual en neonatos se aplican entre los 0 a 30 días de nacidos. En un estudio de histología se reportó que no hay una elongación o ruptura espontanea del frenillo lingual, por lo que su fijación no va variar a lo largo de la vida [16], y de ahí la importancia de un diagnóstico temprano.

Una de las metodologías más usadas es la que propuso Hazelbaker en 1993, llamada "Assessment Tool for Lingual Frenulum Function" (ATLFF) [17], y que fue modificado en 2017 [18]. Está compuesta por dos ítems: el primero, que es anatomía, y el segundo, que es funcionalidad. Esta herramienta cuantitativa marca tres puntuaciones, perfecta, aceptable y función deficiente [19], sugiriendo que el diagnóstico de una anquiloglosia significativa puede determinarse mediante un puntaje total de ocho o menos, y que en tal caso, la frenectomía estaría indicada. Sin embargo, no existe evidencia suficiente que sustente esta aseveración [20]. En la aplicación se han encontrado dificultades con ciertas limitaciones en algunos casos de puntuación y poca utilidad para identificar la severidad de la anquiloglosia [21, 6, 22], puesto que este sistema no evalúa el frenillo lingual posterior [22,23].

Gracias a la ambigüedad del protocolo, este ha tomado nombre dentro del campo de evaluación del frenillo lingual, por lo que es usado en varios estudios de casos, aunque presente detractores [18,24,25]. En el año 2013, CEFAC valido parcialmente el protocolo para la aplicación en neonatos [26,27], y luego lo valida completamente en 2015, por artículos que ha viabilizado su diagnóstico [11].

Otro de los métodos encontrados de evaluación de frenillo lingual en recién nacidos es el tamizaje que propuso Ingram et al. en 2015, Bristol Tongue Assessment Tool (BTAT) [28], que tiene como objetivo facilitar la implementación de los diagnósticos. También se pueden hacer utilizando el juicio clínico subjetivo de los profesionales [29], por lo que no es com- 
pletamente objetivo. A pesar de eso, es una herramienta sencilla con buena capacidad de repetitividad [22], que está compuesta por cuatro ítems, igualmente dividida en dos fases: anatomía y funcionalidad. Este protocolo cumplió parcialmente las normas internacionales del proceso de validación [30]. Al mismo tiempo, se encontró que fue desarrollado en base a la práctica clínica y con referencia a la herramienta de evaluación Hazelbaker "Assessment Tool for Lingual Frenulum Function" (ATLFF) [28].

Finalmente, se halló el test de la lengüita, y que inicialmente fue propuesto por Martinelli et al. en 2012, en base a una revisión de literatura que considera las variaciones anatómicas del frenillo lingual y las funciones de succión y deglución [31], y complementado en 2014, donde se declaró obligatoria la aplicación de este test a todos los recién nacidos de Brasil, por medio de la Ley $\mathrm{N}^{\circ}$ 13.002, 20 de Junio de 2014. [32]. Debido a ese reconocimiento, se convierte en una metodología viable y de alta calidad. De igual manera, se ha sometido a diversos estudios de viabilidad, obteniendo muy buenas calificaciones, con un $100 \%$ en sensibilidad especificidad $[33,34]$, las que concluyeron que la prueba del test de la lengüita demostró ser un instrumento válido y confiable, asegurando la exactitud en el diagnóstico de las alteraciones del frenillo lingual [35]. Todo esto teniendo en cuenta que es el único método de evaluación que considera la historia clínica como un ítem aparte de los anatomo-fucionales. Por consiguiente, México está considerando aplicar el mismo sistema en su país [36,37].

Este test permite una selección inicial llamada triaje neonatal, el cual se realiza en las primeras 48 horas, en el que se tomarán los ítems anatomo-funcionales, permitiendo diagnosticar los casos de mayor severidad. Ahora, si llega a existir una duda, se realiza un retest a los 30 días de nacidos, en el que se valora completamente, considerando que el recién nacido debe adecuarse a los nuevos cambios que enfrenta y que estos toman de 15 a 20 días.

\section{Conclusiones}

1. Según la revisión que se realizó, no se evidencia mayor información actualizada sobre la temática de evaluación de frenillo lingual en neonatos, porque aún no existe claridad en el manejo de esta afección.

2. Necesariamente, el protocolo más usado no es el más eficaz para dar un diagnóstico sobre alguna alteración en el frenillo lingual. Por esa razón, en los estudios se encontró una aplicabilidad alta bajo el método Assessment Tool for Lingual Frenulum Function de Hazelbaker, siendo el mismo que recibe más críticas en los artículos.

3. Según la evidencia, el test de la lengüita es el método más eficaz para evaluar el frenillo lingual en neonatos, porque maneja todos los componentes necesarios para dar un diagnóstico, como lo son los aspectos de historia clínica, anatomía, funcionalidad y lactancia materna.

\section{Referencias}

1. Miranda PP, Cardoso CL, Gomes E. Intervenciones en la alteración del frenillo lingual: revisión sistemática. En t. Arco. Otorrinolaringol. [Internet]. Septiembre de 2016 [consultado el 30 de abril de 2021];20(3):275-280. https://doi.org/10.1055/s-0035-1568135.

2. Pérez NN, López M. Anquiloglosia en niños de 5 a 11 años de edad: Diagnóstico y tratamiento. Rev Cubana Estomatol [Internet]. 2002 Dic [citado 2021 Mayo 14];39(3):282-301. Disponible en: http://scielo.sld.cu/scielo.php?script=sci_arttext\&pi$\mathrm{d}=$ S0034-75072002000300002\&lng $=$ es 
3. Pompéia LE, Ilinsky RS, Ortolani CLF, Faltin JK. A influência da anquiloglossia no crescimento e desenvolvimento do sistema estomatognático. Rev. paul. pediatr. [Internet]. 2017 June [cited 2021 Apr 30];35(2):216-221. DOI: https://doi.org/10.1590/19840462/;2017;35;2;00016

4. Kupietzky A, Botzer E. Anquiloglosia en el lactante y el niño pequeño: sugerencias clínicas para el diagnóstico y el tratamiento. Odontología pediátrica. 2005;27(1):40-46. Disponible en: https://www.ingentaconnect.com/content/aapd/pd/2005/00000027/00000001/ art00009\#expand/collapse

5. Jaque Rocha S, Jeldes Díaz P, Mieres Madrid J. Valoración de movimientos orofaciales en menores de 3 a 4 años con desarrollo normal: datos normativos. Repositorio académico de la Universidad de Chile; 2011. Disponible en: http://repositorio.uchile.cl/ handle/2250/114886

6. Pransky SM, Lago D, Hong P. Breastfeeding difficulties and oral cavity anomalies: The influence of posterior ankyloglossia and upper-lip ties. Int J Pediatr Otorhinolaryngol [nternet]. 2015 Oct [cited 2018 May 30];79(10):1714-7. DOI: https://doi.org/10.1016/j. ijporl.2015.07.033

7. Ferreira González I, Urrútia G, Alonso-Coello P. Revisiones sistemáticas y metaanálisis: bases conceptuales e interpretación. Revista Española de Cardiología. 2011;64(8):688696. DOI: https://doi.org/10.1016/j.recesp.2011.03.029

8. Orte-González EM, Alba-Giménez L, Serrano-Alvar B. La anquiloglosia y las dificultades que presenta en el amamantamiento. Matronas Profesión. 2017;18(3):e50-e57. Disponible en: https://www.federacion-matronas.org/revista/wp-content/uploads/2018/01/ revbibliografica-anquiologlosia.pdf

9. Corrêa MSNP, Abanto Alvarez J, Corrêa FNP, Bonini G, Alves FBT. Anquiloglosia y amamantamiento: Revisión y reporte de caso. REH [Internet]. 16 sep. 2014 [citado 30abr.2021];18(2):123. DOI: https://doi.org/10.20453/reh.v18i2.1844

10. Messner AH, Lalakea ML, Aby J, Macmahon J, Bair E. Ankyloglossia: Incidence and Associated Feeding Difficulties. Arch Otolaryngol Head Neck Surg. 2000;126(1):36-39. DOI: https://doi.org/10.1001/archotol.126.1.36

11. Ballard JL, Auer CE, Khoury JC. Ankyloglossia: Assessment, Incidence, and Effect of Frenuloplasty on the Breastfeeding Dyad. Pediatrics. November 2002;110(5):e63. DOI: https://doi.org/10.1542/peds.110.5.e63

12. Griffiths DM. Do Tongue Ties Affect Breastfeeding? Journal of Human Lactation. 2004;20(4):409-414. DOI: https://doi.org/10.1177/0890334404266976

13. Notestine GE. The Importance of the Identification of Ankyloglossia (Short Lingual Frenulum) as a Cause of Breastfeeding Problems. Journal of Human Lactation. 1990;6(3):113-115. DOI: https://doi.org/10.1177/089033449000600316

14. González Jiménez D, Costa Romero M, Riaño Galán I, González Martínez MT, Rodríguez Pando MC, Lobete Prieto C. Prevalencia de anquiloglosia en recién nacidos en el Principado de Asturias. Anales de Pediatría. August 2014;81(2):115-119. DOI: https:// doi.org/10.1016/j.anpedi.2013.10.030 
15. Carmona Romero MF, Lozada Ramón DS, Romero Pinzón GA. Efecto de una leche comercial suplementada con probióticos sobre factores biológicos asociados a caries dental de niños. Repositorio Institucional Universidad Cooperativa de Colombia. (Tesis de pregrado); 2018. Recuperado de: https://repository.ucc.edu.co/handle/20.500.12494/6629

16. Oliveira YA. Prevalência da anquiloglossia em lactentes: estudo Rretrospectivo. Trabalho de Conclusão de Curso (Graduação em Odontologia). Repositório Institucional - Universidade Federal de Uberlândia; 2017. Disponible en: https://repositorio.ufu.br/handle/123456789/19427

17. Hazelbaker AK. The Assessment Tool for Lingual Frenulum Function (ATLFF): Use in a Lactation Consultant Private Practice. [Thesis]. Pasadena, CA: Pacific Oaks College; 1993.

18. Hazelbaker AK. Assessment Tool for Lingual Frenulum Function (ATLFF). Clinical Lactation [Internet]. Springer Publishing Company; 2017;8(3):132-3. Doi: http://dx.doi. org/10.1891/2158-0782.8.3.13

19. Aguilar Cañas S, Llanos Redondo A, Ayala Nieto A, Portilla Portilla E, Espinal Rangel R. Metodologia: tamiz auditivo neonatal. Revista Signos Fónicos. 2016;2(3):161-172. DOI: https://doi.org/10.24054/01204211.v3.n3.2016.2002

20. Robles-Andrade MS, Guerrero-Sierra C. Impacto de la anquiloglosia y la frenotomía lingual en la alimentación neonatal. Perinatol. Reprod. Hum. [Internet]. 2014 Sep [citado 2021 Mayo 14];28(3):154-158. Disponible en: http://www.scielo.org.mx/scielo. php?script=sci_arttext\&pid=S0187-53372014000300006\&lng=es.

21. Ricke LA, Baker NJ, Madlon-Kay DJ, DeFor TA. Newborn tongue-tie: prevalence and effect on breast-feeding. J Am Board Fam Pract. 2005 Jan-Feb;18(1):1-7. DOI: https:// doi.org/10.3122/jabfm.18.1.1

22. Aguilar Cañas S, Crespo Pérez N, Mejía Martínez N, Ramírez Arias L, Tamara Moncada V. Ruta de atención, criterios y metodologías para atención de lactantes con anquiloglosia. Revista Signos Fónicos. 2018;4(2). DOI: https://doi.org/10.24054/01204211. v2.n2.2018.3971

23. O'Shea JE, Foster JP, O'Donnell CP, Breathnach D, Jacobs SE, Todd DA, Davis PG. Frenotomy for tongue-tie in newborn infants. Cochrane Database Syst Rev. 2017 Mar 11;3(3). DOI: https://doi.org/10.1002/14651858.CD011065.pub2

24. Lopes R, Martinelli DC, Marchesan IQ. Protocol for Infants: Relationship Between Anatomic and Functional Aspects. Revista CEFAC. 2013;15(3):599-609. DOI https://doi. org/10.1590/S1516-18462013005000032

25. Martinelli RL, Marchesan IQ, Berretin-Felix G. Lingual frenulum protocol with scores for infants. Int J Orofacial Myology. 2012 Nov;38:104-12. DOI: http://www.abramofono.com.br/wp-content/uploads/2012/02/Lingual-Frenulum-Protocol-with-scores-for-infants-IJOM-2012.pdf

26. Fernández-Dávila M. Características en la producción del habla en niños de 6 a 7 años con frenillo lingual alterado. Revista Signos Fónicos.2015; 1(2). DOI: https://doi. org/10.24054/01204211.v2.n2.2015.1731 
27. Amir L, James J, Donath S Reliability of the hazelbaker assessment tool for lingual frenulum function.. Int Breastfeed J. 2006;1(1):3 DOI: https://doi.org/10.1186/1746-4358-1-3

28. Ingram J, Johnson D, Copeland M, Churchill C, Taylor H, Emond A. The development of a tongue assessment tool to assist with tongue-tie identification. Archives of Disease in Childhood - Fetal and Neonatal Edition. 2015;100(4):F344-F349. Disponible en: https://fn.bmj.com/content/100/4/F344.short

29. O'Shea JE, Foster JP, O’Donnell CP, Breathnach D, Jacobs SE, Todd DA, et al. Frenotomy for tongue-tie in newborn infants. Cochrane Database Syst Rev [Internet]. 2017 Mar 11 [cited 2018 May 30];3:CD01 1065. DOI: https://doi.org/10.1002/14651858. CD011065.pub2

30. Lopes de Castro Martinelli RL, Marchesan IQ, Lauris JR, Honório HM, Gusmão RJ, Berretin-Felix G. Validade e confiabilidade da triagem: "teste da linguinha". Rev. GEFAC [Internet]. 2016 Dec [cited 2021 Apr 30];18(6):1323-1331. DOI: https://doi. org/10.1590/1982-021620161868716

31. Lopes de Castro Martinelli R, Marchesan IQ, Berretin-Felix G. Lingual frenulum evaluation protocol for infants: relationship between anatomic and functional aspects. Rev. CEFAC. 2013 Mai-Jun;15(3):599-610. Available from: https://www.scielo.br/pdf/rcefac/2013nahead/en_162-11.pdf

32. Lopes de Castro Martinelli R, Jordão Gusmão R, Moya Daza MP, Queiroz Marchesan I, Berretin-Felix G. Profile of Scientific Production on Ankyloglossia. Int J Med Surg Sci [Internet]. 2020;8(1):1-13.. Disponible en: https://pesquisa.bvsalud.org/portal/resource/pt/biblio-1151622

33. de Almeida KR, Leal TP, Kubo H, Castro TES, Ortolani CLF. 'Lingual frenotomy in a newborn, from diagnosis to surgery: a case report', Revista CEFAC: Atualizacao Cientifica em Fonoaudiologia e Educacao. 2018;20(2):258.DOI: https://dx.doi. org/10.1590/1982-0216201820212917

34. Das Graças Mosele P, Ferreira dos Santos J, Cristina de Godói V, Marson Costa F, Marco De Toni P, Ide Fujinaga C. Assessment Scale of Newborn Sucking for Breastfeeding. Revista CEFAC. Set-Out. 2014;16(5):1548-57. DOI: https://doi.org/10.1590/19820216201426412

35. Lopes de Castro Martinelli R. Validação do protocolo de avaliação do frênulo da língua em bebês. Biblioteca digital Universidade de São Paulo; 2016. DOI: https://doi. org/10.11606/T.25.2016.tde-17052016-150210

36. Todd DA. Tongue-tie in the newborn: what, when, who and how? Exploring tongue-tie division. Breastfeed Rev [Internet]. 2014 Jul [cited 2018 May 30];22(2):7-10. Disponible en: https://search.informit.org/doi/10.3316/INFORMIT.598687183131831

37. Llanos Redondo A, Rangel Navia HJ, Aguilar Cañas SJ, Tamara Moncada VA, Crespo Pérez NC. Evaluación de anquiloglosia y lactancia materna en lactantes de 0 a 24 meses. Revista Salud Bosque. 2020;10(2). DOI: https://doi.org/10.18270/rsb.v10i2.3015 